\title{
Ecocardiografía funcional como una herramienta de evaluación dinámica del tratamiento en neonatos críticamente enfermos
}

\author{
Functional echocardiography as a tool for the dynamic treatment evaluation \\ in critically ill neonates
}

\author{
Ana L. Acevedo-Olguín ${ }^{1 *}$, Rodrigo Hernández-Benítez ${ }^{2}$, José Iglesias-Leboreiro ${ }^{3}$, \\ Isabel Bernárdez-Zapata ${ }^{3}$, Moisés N. Gerardo-del Hoyo y Mario E. Rendón-Macías ${ }^{4}$ \\ ${ }^{1}$ Neonatología; 2 Departamento de Pediatría, Cardiología pediátrica; ${ }^{3}$ Departamento de Neonatología. Hospital Español de México; ${ }^{4}$ Unidad de \\ Investigación en Análisis y Síntesis de la Evidencia, IMSS, Ciudad de México, México
}

\begin{abstract}
Resumen
Introducción: Aún existe controversia sobre la utilidad de la ecocardiografía funcional (EcoFn) en la valoración cardiovascular de neonatos críticamente enfermos. Objetivo: Analizar la utilidad de la EcoFn en la modificación de tratamiento en neonatos ingresados a una Unidad de Cuidados Intensivos Neonatales (UCIN). Material y métodos: Estudio prospectivo en una UCIN durante un año mediante EcoFn realizada por un cardiólogo capacitado en pacientes en sus primeras 72 horas de vida extrauterina, con valoración de la funcionalidad cardiaca y presencia de alteraciones estructurales. Con base en los hallazgos se analizaron las modificaciones al tratamiento. Resultados: 37 neonatos fueron evaluados con EcoFn en dos ocasiones: una diagnóstica y otra de seguimiento. En 11 (29.7\%) se encontró daño estructural y funcional, en 9 (24.3\%) solo funcional, en 7 (18.9\%) solo estructural y en 10 (27\%) no se observaron alteraciones. En el 70\% se realizó al menos un cambio de tratamiento. Los cambios más frecuentes fueron modificaciones al apoyo ventilatorio (63\%), seguido del apoyo aminérgico (19.5\%) y modificaciones en el aporte de líquidos (10.8\%). En todos los casos los cambios mejoraron las condiciones clínicas de los neonatos. Conclusiones: La EcoFn permitió determinar mejor las condiciones estructurales y hemodinámicas de los pacientes y realizar modificaciones terapéuticas más precisas.
\end{abstract}

Palabras claves: Neonato. Pretérmino. EcoFn. Ucin. Tratamiento. México.

\begin{abstract}
Introduction: It is still controversy about the usefulness of functional echocardiography (FnEC) in critically ill neonates. Objective: To analyze the usefulness of the FnEC in the treatment decisions in neonates admitted to a Neonatal Intensive Care Unit (NICU). Materials and methods: A year prospective study in a NICU. A trained cardiologist performed a FnEC to $72 \mathrm{~h}$ of life patients to evaluate the cardiac function and heart structure. We analyzed the clinical decisions after the findings. Results: 37 neonates underwent two FnEC: at the diagnosis and at follow-up. Eleven patients (29.7\%) had structural and functional abnormalities, 9 (24.3\%) only functional, 7 (18.9\%) only structural, and in the rest, 10 (27\%), were normal. Al least
\end{abstract}

Correspondencia:

*Ana Laura Acevedo-Olguín

E-mail: acevedolguin@gmail.com
Disponible en internet: 09-12-2019 Arch Cardiol Mex. 2019;89(4):393-398 www.archivoscardiologia.com 1405-9940/@ 2019 Instituto Nacional de Cardiología Ignacio Chávez. Publicado por Permanyer. Este es un artículo open access bajo la licencia CC BY-NC-ND (http://creativecommons.org/licenses/by-nc-nd/4.0/) 
one change in the management occurred in $70 \%$ of the patients. The main changes were to the ventilatory support (63\%), followed by inotropic support (19.5\%) and fluid intake (10.8\%). In all changes, we observed an improvement in the clinical conditions of the neonates. Conclusions: The FnEC allowed to determinate the cardiovascular structures and hemodynamic conditions of the patients and make a more precise therapeutic modifications.

Key words: Neonate. Preterm. EcoFn. Treatment. NICU. Mexico.

\section{Introducción}

La evaluación clínica continua del estado hemodinámico en un neonato críticamente enfermo es fundamental para realizar acciones dirigidas a preservar la perfusión y oxigenación tisular, principalmente en los órganos vitales ${ }^{1}$. Dicha evaluación suele realizarse por medio del registro de la frecuencia cardiaca, presión arterial, el llenado capilar y el gasto urinario², variables que deben ser analizadas considerando los cambios asociados a la edad gestacional al nacimiento ${ }^{3}$. Aunado a lo anterior, la medición de estas variables puede ser difícil, sobre todo si no se cuenta con equipos especiales y/o no se desea recurrir a un monitoreo invasivo ${ }^{4}$. Por ello se han buscado otras alternativas menos agresivas y más fiables para esta valoración.

La ecocardiografía funcional (EcoFn) se introdujo aproximadamente hace 10 años como una herramienta de apoyo para la valoración hemodinámica continua de los neonatos críticamente enfermos. Por medio de esta es factible: a) dar evidencia de alteraciones estructurales del corazón y grandes vasos, tales como cortocircuitos intracardiacos y extracardiacos; b) evaluar la función miocárdica (volúmenes de llenado y vaciamiento ventricular y tiempos de contracción), y c) determinar las presiones de flujos sistémico y pulmonar².

La valoración de la precarga se realiza a través de la valoración del ventrículo izquierdo $(\mathrm{VI})$, ventrículo derecho (VD) y la vena cava inferior (VCl), en caso de urgencias el observar el signo de «beso entre los ventrículos" sugiere hipovolemia, la dilatación del VD puede indicar sobrecarga o presencia de hipertensión arterial pulmonar (HAP); siempre es importante recordar que en neonatos el VD es ligeramente dominante tras el nacimiento.

La complejidad dinámica de la circulación transicional, la respuesta que puede tener un miocardio inmaduro en las etapas tempranas del periodo neonatal, la existencia de cortocircuitos fisiológicos tales como el foramen oval y la persistencia de conducto arterioso (PCA), hacen que la decisión terapéutica sea un reto. El rol de cardiólogo en la Unidad de Cuidados Intensivos Neonatales (UCIN) debe enfocarse no solo a la parte anatómica sino también a la hemodinámica, y debe de ser de forma continua; diversos estudios muestran que el uso de la EcoFn de forma sistemática permite identificar compromiso cardiovascular, permitiendo al neonatólogo realizar cambios en el manejo y mejorar a corto plazo la evolución de los pacientes ${ }^{3,5}$.

Se ha incrementado el espectro de indicaciones hasta convertirse prácticamente en una prolongación de la exploración física; se suma a la valoración de los datos clínicos habituales, en un intento de individualizar los tratamientos en función de la fisiopatología concreta en cada situación $n^{3}$.

Una ventaja potencial de esta técnica es su carácter dinámico, con ello facilita la toma de decisiones terapéuticas fundadas en las condiciones fisiopatológicas de cada paciente, además de observar los cambios consecuentes a estas modificaciones ${ }^{3,6}$. Existen diversos estudios a nivel mundial en los cuales se reportan cambios en el tratamiento clínico en un 30-60\% de los casos, en algunos hospitales este tipo de estudio se considera indispensable en el manejo del neonato con choque $^{5,6}$.

Una desventaja es su característica de ser dependiente del operador, ya que su precisión depende de la capacitación del cardiólogo evaluador ${ }^{4}$. En nuestro hospital esta técnica ha sido implementada hace cinco años y al momento no se ha evaluado su impacto en la atención de nuestros neonatos.

El objetivo de este estudio es informar sobre la utilidad de la EcoFn en el manejo hemodinámico de neonatos críticamente enfermos de una UCIN.

\section{Material y métodos}

Estudio descriptivo de una cohorte prospectiva de neonatos atendidos en la UCIN del Hospital Español de México. Estudio aprobado por el Comité de investigación y ética del Hospital Español.

Se incluyeron neonatos pretérmino y término en cualquier momento de su estancia en la UCIN atendidos durante el periodo de 1 de marzo del 2017 al 28 de febrero del 2018. Durante el tiempo del estudio, diariamente se evaluaron a los neonatos hospitalizados para detectar aquellos que tuvieran alguno de los siguientes datos: 
- Datos de dificultad respiratoria persistente a pesar del manejo ventilatorio establecido.

- Datos de inestabilidad hemodinámica (hipotensión, taquicardia o bradicardia, oliguria, Ilenado capilar prolongado).

- Antecedentes maternos con riesgo de repercusión cardiaca (madres con lupus eritematoso sistémico [LES]).

- Evidencia de malformaciones congénitas extracardiacas y sospecha de cardiopatía congénita.

Una vez aprobada la participación por los padres o tutores responsables, se solicitó la valoración por el Servicio de Cardiología para EcoFn.

El estudio se realizó en la cuna del paciente, por medio de un ultrasonido Siemens Acuson X150 Vet ${ }^{\circledR}$ con transductor $\mathrm{S} 8^{\circledR}$ de 2.25 a $12 \mathrm{MHz}$. Las mediciones se realizaron por un solo observador, cardiólogo pediatra ecocardiografista con cinco años de experiencia en esta técnica.

La valoración ecocardiográfica se dirigió principalmente a los siguientes aspectos:

- Valorar la persistencia de conducto arterioso y su repercusión (diámetro de la boca pulmonar, velocidad ductal, radio entre aurícula izquierda (Al) y raíz aórtica, E/A mitral y dirección del cortocircuito).

- Valorar la función sistólica y diastólica del VI. Se estimo la fracción de acortamiento (FA) y la de eyección (FEVI) en modo $M$ en vistas paraesternales en eje corto y eje largo o utilizando el método biplano de Simpson. Los valores que se tomaron como normales fueron: FA entre el 26 y el $46 \%$, FEVI normal > 55\%. Doppler tisular: se determinó el índice de rendimiento miocárdico (TEI), el cual ha demostrado una adecuada correlación con la contractilidad global y regional ventricular. Se consideraron valores normales entre 0.280.32 para ambos ventrículos.

- Función ventricular derecha. Tricuspid Annular Plane Systolic Excursion (TAPSE). Se determino por medio de modo $\mathrm{M}$ a través del anillo lateral tricuspídeo, midiendo el movimiento longitudinal del anillo tricuspídeo en sístole. Es un buen parámetro de función sistólica.

- Estimación de la presión sistólica de la arteria pulmonar (PSAP). Se estimó desde una vista de cuatro cámaras para obtener un buen trazo de la insuficiencia tricuspídea, a mediante Doppler CW; posteriormente se agregó la presión auricular derecha, que es normalmente de entre $5-10 \mathrm{mmHg}$.

Fórmula de PSAP $=4 \times($ velocidad de la IT $) 2+$ presión de la $A D(5-10 \mathrm{mmHg})$.

En caso de tener flujo en el conducto arterioso, esta se determinó con base en su gradiente menos la presión arterial sistólica.
- Determinar la presencia de comunicación interauricular.

- Estimación del gasto sistémico y pulmonar.

- Estimación del gasto sistémico. Se realizó mediante el área seccional (CSA), la cual se obtuvo midiendo el diámetro en el sitio de inserción de las valvas aórticas al final de la sístole. La integral velocidad tiempo (VTI) se midió con Doppler PW a nivel proximal de la válvula aórtica en una vista apical de cinco cámaras.

Gasto sistémico $(\mathrm{ml} / \mathrm{kg} / \mathrm{min})=$ CSA $\times$ VTI - medición tracto de salida del VI x FC/peso $(\mathrm{kg})$

- Estimación del gasto pulmonar. Se realizo mediante el CSA, la cual se obtuvo midiendo el diámetro en el sitio de inserción de las valvas pulmonares al final de la sístole. La VTI se midió con Doppler PW a nivel proximal de la válvula pulmonar en una vista de eje corto.

Gasto pulmonar $(\mathrm{ml} / \mathrm{kg} / \mathrm{min})=\mathrm{CSA} \times \mathrm{VTI}-$ medición tracto de salida del VD x FC/peso (kg)

- Determinar el incremento o la disminución del aporte de líquidos. Se determinó con base en mediciones de la Al en comparación con la aorta (Ao), además de una vista de cuatro cámaras, un índice Al: Ao > 1.4:1 en pacientes con PCA indica datos de sobrecarga. Índice de colapsabilidad de la VCl: el colapso de la $\mathrm{VCl}$ a través de los ciclos respiratorios es un buen indicador de precarga en pacientes pediátricos.

- Medir flujo de la vena cava superior (VCS) (diámetro de la VCS y VTI).

En todas las evaluaciones el cardiólogo estuvo enterado de los diagnósticos y tratamientos recibidos por los pacientes. Posteriormente a la valoración y con base en los hallazgos, este sugirió recomendaciones de manejo para mantener o mejorar las condiciones hemodinámicas del neonato. La decisión final de realizarlas fue del neonatólogo responsable de la atención del paciente. Una segunda valoración con EcoFn se realizó a las 72 horas para determinar si hubo cambios asociados al manejo.

De cada neonato se obtuvo información sobre su edad gestacional al nacer, su sexo, los días de vida al estudio, los diagnósticos al momento del estudio y el motivo de solicitud de la EcoFn y el tratamiento recibido al momento de la evaluación.

\section{Análisis estadístico}

Para determinar mejor la utilidad de la EcoFn, los pacientes fueron clasificados después de la valoración en cuatro grupos: I) con corazón estructural y funcionalmente normal; II) con corazón estructuralmente 
anormal y funcionalmente normal; III) con corazón estructuralmente normal pero funcionalmente anormal, y IV) corazón estructural y funcionalmente anormal. Según los hallazgos se resumieron las decisiones consideradas por el ecocardiólogo en: 1) cambios en el requerimientos de líquidos, 2) apoyo de fármacos aminérgicos, 3) manejo ventilatorio, y 4) uso de diuréticos. Se realizó un análisis descriptivo con frecuencias simples y relativas en porcentaje. El análisis estadístico se realizó con apoyo del programa estadístico SPSS ${ }^{\circledR} 14$.

\section{Resultados}

Se estudió a 37 neonatos, como se muestra en la (Tabla 1), tuvimos más pacientes masculinos y el grupo de edad más frecuente fue el de prematuros tardíos (el $79.4 \%$ fueron prematuros), en cuanto al peso tuvimos pacientes por debajo de $1 \mathrm{~kg}$ hasta arriba de los $2.5 \mathrm{~kg}$. La valoración EcoFn se realizó en promedio a los dos días de vida. En esta misma tabla se muestran los diagnósticos por los cuales los neonatos estaban siendo atendidos en la UCIN, siendo el síndrome de dificultad respiratoria el principal motivo.

En todos los pacientes se pudo realizar el estudio completo, con una variación en la ejecución de entre 15 y 20 minutos.

En 27 pacientes (72.9\%) se pudo evidenciar al menos un dato anormal. Los principales hallazgos se presentan en la tabla 2. La anomalía estructural más frecuente fue la evidencia de un conducto arterioso permeable $(48.6 \%)$, seguida de la comunicación interventricular (8.1\%). En cuanto a la función cardiaca predominó la evidencia de hipertensión pulmonar (35.1\%) seguido de la sobrecarga de volumen (10.8\%). Es de resaltar que en un paciente el estudio dio evidencia de endocarditis como la causa de la inestabilidad hemodinámica.

En la tabla 3 se muestran recomendaciones emitidas para el manejo con base en los hallazgos de la EcoFn. En los 10 pacientes en los cuales la EcoFn mostró normalidad estructural y funcional esto permitió en el $70 \%$ sugerir la disminución en el apoyo ventilatorio ante la mejoría de los pacientes, y en el restante $30 \%$ continuar con el manejo establecido.

Cuando solo se evidenció daño estructural (9 pacientes), dada la alta frecuencia con persistencia de conducto arterioso las recomendaciones fueron dirigidas a la modificación del apoyo ventilatorio para mejorar la oxigenación con la finalidad de favorecer el cierre. Solo en un paciente además se sugirió modificar el aporte de líquidos.
Tabla 1. Características de los neonatos estudiados

\begin{tabular}{|l|c|}
\hline Características & Total $37(\%)$ \\
\hline Sexo & $14(37.8)$ \\
\hline Memenino & $23(62.2)$ \\
\hline Semanas de gestación & \\
\hline$<28$ & \\
$28-33$ & $3(8.1)$ \\
$34-36$ & $11(29.7)$ \\
$37-41$ & $15(40.5)$ \\
\hline Peso al nacimiento & $8(21.6)$ \\
\hline 1 kg & \\
$1-1.5$ kg & $4(10.8)$ \\
$1.5-1.990$ kg & $6(16.2)$ \\
$2-2.499$ kg & $8(21.6)$ \\
$>2.5$ & $6(16.2)$ \\
\hline Días de vida al momento de estudio & $13(35.1)$ \\
Mediana & \\
\hline (mín-01-03-máx) & 2 \\
\hline Diagnóstico principal & $(1-1-3-30)$ \\
\hline Síndrome de dificultad respiratoria & \\
Taquipnea transitoria del recién nacido & $20(54.1)$ \\
Sepsis & $4(10.8)$ \\
Prematurez extrema & $3(8.1)$ \\
Hidrops fetalis & $3(8.1)$ \\
Hijo de madre con LES & $2(5.4)$ \\
Hernia diafragmática & $2(5.4)$ \\
Atresia esofágica & $1(2.7)$ \\
Asfixia perinatal & $1(2.7)$ \\
& $1(2.7)$ \\
\hline
\end{tabular}

LES: lupus eritematoso sistémico.

Tabla 2. Hallazgos ecocardiográficos en 37 neonatos por EcoFn

\begin{tabular}{|l|c|}
\hline $\begin{array}{l}\text { Hallazgos ecocardiográficos en } 37 \\
\text { neonatos por EcoFn }\end{array}$ & Total \\
\hline Hallazgos en EcoFn & $\mathbf{n}(\%)$ \\
\hline Persistencia conducto arterioso & $18(48.6)$ \\
\hline HAP & $13(35.1)$ \\
\hline Sobrecarga de volumen & $4(10.8)$ \\
\hline CIV minúscula & $3(8.1)$ \\
\hline Persistencia de circulación fetal & $1(2.7)$ \\
\hline Bajo gasto cardiaco & $1(2.7)$ \\
\hline Insuficiencia tricuspídea & $1(2.7)$ \\
\hline Endocarditis & $1(2.7)$ \\
\hline
\end{tabular}

Nota: algunos pacientes tuvieron más de un hallazgo en el estudio. EcoFn: ecocardiografía funcional; HAP: hipertensión arterial pulmonar; CIV: comunicación interauricular.

En los pacientes en donde había repercusión hemodinámica (siendo definida como gastos cardiacos por debajo de los valores normales, insuficiencias valvulares 
Tabla 3. Decisiones de manejo posterior a la valoración por ecocardiografía funcional (EcoFn)

\begin{tabular}{|c|c|c|c|c|}
\hline \multicolumn{5}{|c|}{ Decisiones de manejo posterior a la valoración por EcoFn } \\
\hline & $\begin{array}{l}\text { Estructura y función } \\
\text { normal } \\
(n=10)\end{array}$ & $\begin{array}{l}\text { Daño estructural } \\
\text { función normal } \\
\qquad(\mathrm{n}=9)\end{array}$ & $\begin{array}{l}\text { Estructura normal } \\
\text { función alterada } \\
\qquad(\mathbf{n}=7)\end{array}$ & $\begin{array}{l}\text { Daño estructural y } \\
\text { función alterada } \\
\text { ( } n=11)\end{array}$ \\
\hline Aumento del aporte de líquidos & & & $1(14.2 \%)$ & \\
\hline Inicio de apoyo aminérgico & & & $3(42.8 \%)$ & $5(45.4 \%)$ \\
\hline Suspensión del apoyo aminérgico & & & $1(14.2 \%)$ & \\
\hline Incremento en el apoyo ventilatorio & & $8(88.8 \%)$ & $6(85.7 \%)$ & $4(36.3 \%)$ \\
\hline Disminución del apoyo ventilatorio & $7(70 \%)$ & $2(22.2 \%)$ & & $2(18.1 \%)$ \\
\hline Disminución de aporte de líquidos & & $1(11.1 \%)$ & $3(42.8 \%)$ & $1(9 \%)$ \\
\hline Inicio de diuréticos & & & $2(28.5 \%)$ & \\
\hline Total de cambios & 7 & 11 & 16 & 12 \\
\hline
\end{tabular}

Nota: en un paciente se pudo realizar más de un cambio en su manejo.

de moderadas a graves, HAP o FEVI y FA por debajo de los valores normales) en corazones estructuralmente normales (7 pacientes) el estudio permitió realizar diferentes modificaciones tanto en manejo ventilatorio, apoyo aminérgico y aporte de líquidos. Lo más frecuente fue incremento en la asistencia ventilatoria, apoyo aminérgico y restricción del aporte de líquidos.

Por último, en los pacientes con disfunción cardiaca en corazones con alguna alteración estructural (11 pacientes), la EcoFn permitió sugerir cambios específicos en la ventilación, apoyo aminérgico y en el manejo de líquidos. Cinco pacientes presentaron una $\mathrm{FEVI}<55 \%$, con presencia de alargamiento de los tiempos de relajación y contracción isovolumétrica, reflejando un TEI alargado (> 0.32); en estos pacientes se sugirió inicio de aminas, en un caso se sugirió también el incremento del apoyo ventilatorio, en un caso se observó una VCI dilatada, con crecimiento de cavidades derechas, insuficiencia tricuspídea y pulmonar de leves a moderadas, con lo cual se indicó la disminución de líquidos. En tres casos se sugirió el incremento del apoyo ventilatorio por datos de disfunción ventricular derecha, presencia de IT moderada a grave, un TAPSE bajo, alteración del TEI del VD o desplazamiento del tabique interventricular. Por último, en dos casos se sugirió la disminución del apoyo ventilatorio, ya que presentaban una función ventricular derecha adecuada, presentaban PCA pero con leve repercusión en la función ventricular izquierda.

En todos los casos se pudo evidenciar que a las 72 horas el control por EcoFn mostró mejoría en la función cardiovascular.

\section{Discusión}

En las últimas dos décadas, la incorporación de la EcoFn como una herramienta no invasiva en el diagnóstico de las condiciones estructurales y funcionales de los neonatos críticamente enfermos en terapias intensivas ha incrementado. Su utilidad, sin embargo, ha sido poco evaluada y por ello este trabajo muestra nuestra experiencia en su uso durante un año. Como se mostró en los resultados, podemos afirmar que en prácticamente todos nuestros pacientes, la EcoFn aportó información útil para el manejo. En el $70 \%$ de ellos directamente aportó datos para cambios al tratamiento y en el 30\% restante confirmó la adecuación del manejo ya establecido.

Dos aspectos que aporta nuestro estudio son:

- Por un lado la decisión de la realización de las EcoFn como un procedimiento habitual en todo neonato críticamente enfermo, contrario a lo observado en estudios previos ${ }^{3,7}$, donde la indicación de la EcoFn ha sido sobre todo ante dudas en el diagnóstico y tratamiento del conducto arterioso, o en neonatos con inestabilidad hemodinámica ${ }^{10,12}$. Esta diferencia bien puede explicar el mayor número de modificaciones al manejo en nuestro estudio $(70 \%)$ frente a las informadas, del 30 al 51\%7. En nuestro estudio la valoración tiene un objetivo tanto de diagnóstico estructural como funcional.

- El otro aspecto diferente es la realización por un cardiólogo pediatra. Esta aportación tiene sus ventajas, al mejorarse la precisión diagnóstica estructural y 
funcional, dada la mayor capacitación de este médico. Una de las críticas comunes al uso de la EcoFn es la posibilidad de errores diagnósticos, sobre todo estructurales, cuando un neonatólogo la realiza ${ }^{4,8}$. Por otro lado, el ser llevada a cabo por un cardiólogo tiene la limitante de la disponibilidad de experto; un interés mundial en la incorporación de esta herramienta en la atención cotidiana y dinámica de los neonatos críticamente enfermos ha sido la posibilidad de ser realizada por los propios neonatólogos. Por ello, tanto en EE.UU. como en países europeos se han generado cursos 0 programas de capacitación certificados ${ }^{6,9,13}$.

Nuestro trabajo tiene aún limitaciones a considerarse, la primera es su carácter observacional, con lo cual no podemos afirmar si las modificaciones o su no realización hubieran acontecido igual sin el estudio de Eco$\mathrm{Fn}^{10,11}$. Nosotros recomendamos a futuro un estudio que compare la utilización sistemática de la EcoFn con otra indicada bajo condiciones de inestabilidad del neonato.

Por otro lado, nuestro estudio proporciona información sobre el posible curso de atención en pacientes a quienes la EcoFn se realizó sistemáticamente en una primera valoración cuando el recién nacido aún puede presentar vulnerabilidad cardiocirculatoria, como la inmadurez del miocardio, la presencia de cortocircuitos fetales, las variaciones en las resistencias vasculares pulmonares y sistémicas y, en definitiva, los complejos cambios hemodinámicos que se producen durante el proceso de transición a la vida extrauterina.

En otros estudios lo han evaluado con mediciones sucesivas. En el estudio de Khakmar, et al. ${ }^{7}$ en promedio a los pacientes se les realizaron dos valoraciones $y$ en el estudio de Corredera, et al. hasta un promedio de tres $^{3}$. Por ello, igualmente a futuro se debe considerar la utilidad de una valoración inicial, donde el aspecto estructural será primordial y estudios repetidos funcionales a medida del requerimiento de cada pacientes.

Todavía no existen evidencias firmes que demuestren un impacto positivo de la utilización de la EcoFn en la UCIN sobre el pronóstico de los pacientes; son necesarios más estudios aleatorizados para aclarar si la EcoFn modifica la evolución de los pacientes, así como de la evaluación del costo-efectividad de la implementación del EcoFn de forma sistemática como una herramienta dentro de la UCIN para la supervicencia y condición de salud al egreso de los pacientes.

\section{Conclusión}

La EcoFn mostró ser un estudio seguro y no invasivo para determinar mejor la estructura cardiovascular y las condiciones hemodinámicas de los neonatos. Con la información obtenida es posible apoyar al neonatólogo en la toma de decisiones terapéuticas oportunas y adecuadas.

\section{Financiación}

El autor declara que este trabajo no ha recibido ninguna fuente de financiación externa.

\section{Conflicto de intereses}

Ninguno.

\section{Responsabilidades éticas}

Protección de personas y animales. Los autores declaran que para esta investigación no se han realizado experimentos en seres humanos ni en animales.

Confidencialidad de los datos. Los autores declaran que han seguido los protocolos de su centro de trabajo sobre la publicación de datos de pacientes.

Derecho a la privacidad y consentimiento informado. Los autores declaran que en este artículo no aparecen datos de pacientes.

\section{Bibliografía}

1. Hernández R, Becerra R. Ecocardiografía funcional en cuidados intensivos neonatales: experiencia en un hospital de tercer nivel. Bol Med Hosp Infant Mex. 2016;73(5):325-30.

2. Arruza $L$, Corredera $A$. Ecocardiografía funcional en neonatología. An Pediatr Contin. 2014;12(2):78-84.

3. Corredera A, Rodríguez MJ, Arévalo P, Llorente B, Moro M, Arruza L. Ecocardiografía funcional en cuidados intensivos neonatales: experiencia en una unidad española a lo largo de un año. An Pediatría. 2014; 81(3):167-73.

4. Tissot C, Muehlethaler V, Sekarski N. Basics of functional echocardiography in children and neonates. Front Pediatr. 2017;5:235.

5. Moss S, Kitchiner DJ, Yoxall CW, Subhedar NV. Evaluation of echocardiography on the neonatal unit. Arch Dis Child Fetal Neonatal Ed. 2003;88(4):F287-9; discussion F290-1.

6. Kluckow M, Seri I, Evans N. Functional echocardiography: an emerging clinical tool for the neonatologist. J Pediatr. 2007;150(2):125-30.

7. Khamkar A, Pradeep S, Maheswari R, Patnaik S, Malshe N, Kalrao V, et al. Functional neonatal echocardiography: Indian experience. J Clin Diagn Res. 2015;9(12):SC11-4.

8. Mertens L, Seri I, Marek J, Arlettaz R, Barker P, McNamara P, et al. Targeted neonatal echocardiography in the neonatal intensive care unit: Practice Guidelines and Recommendations for Training: Writing group of the American Society of Echocardiography (ASE) in collaboration with the European Association of Echocardiography (EAE) and the Association for European Pediatric Cardiologists (AEPC). Eur J Echocardiogr. 2011;12(10):715-36.

9. Singh Y. Echocardiographic evaluation of hemodynamics in neonates and children. Front Pediatr. 2017;5:201.

10. Noritomi DT, Vieira ML, Mohovic T, Bastos JF, Cordioli RL, Akamine N, et al. Echocardiography for hemodynamic evaluation in the intensive care unit: Shock. 2010;34(Suppl 1):59-62.

11. Rolland A, Shankar-Aguilera S, Diomandé D, Zupan-Simunek V, Boileau $P$. Natural evolution of patent ductus arteriosus in the extremely preterm infant. Arch Dis Child Fetal Neonatal Ed. 2015;100(1):F55-8.

12. Arlettaz R. Echocardiographic evaluation of patent ductus arteriosus in preterm infants. Front Pediatr. 2017;5:147.

13. Nguyen J, Amirnovin R, Ramanathan R, Noori S. The state of point-of-care ultrasonography use and training in neonatal-perinatal medicine and pediatric critical care medicine fellowship programs. J Perinatol. 2016;36(11):972-6. 